\title{
The Importance and Quality of Personalized Care after Breast Cancer Surgery
}

\author{
Yao Danyang ${ }^{*}$, Zhang Guangying and Wang Bo \\ Jilin Cancer Hospital, Changchun,Jilin 130021, China
}

\begin{abstract}
Objective: To investigate the effect of personalized care after breast cancer surgery. Methods: 70 cases of breast cancer patients in our hospital were selected from the duration of December 2012 until December 2014. The patients were randomly divided into observation group and control group, where each group consists of 35 cases. The control group received routine professional nursing care during perioperative of breast cancer surgery. The observation group received routine professional nursing care and personalized nursing intervention during perioperative of breast cancer surgery. Comparison of two groups of patients were measured by treatment compliance, nursing satisfaction, self-rating anxiety scale and self-rating depression scale evaluation of the psychological state of the patient. Results: Observation group patient compliance and satisfaction were significantly better than the control group, as shown by statistically significant difference between groups $(p<0.05)$. The observation group of SAS and SDS scores improvement were significantly better than the control group, a statistically significant difference between groups $(p<0.05)$. Conclusion: Personalized nursing intervention can improve patient compliance, improve patient's mental state, and worthy of health promotion.
\end{abstract}

\author{
KEYWORDS \\ Personalized nursing \\ Mammary gland \\ Compliance \\ Anxiety \\ Depression
}

\section{Introduction}

During the treatment in breast surgery department, most of the time, it will be a stressful events for patients. It gives patients certain psychological effect, which will not only affect their living conditions, but also the recovery process. Develop individualized care measures according to the psychological characteristics of patients, it can effectively relieve the patient's adverse psychological and improve prognosis [1]. From December 2012 to December 2014, my department took part in breast cancer surgery department personalized care, and the results reported as below.

\section{Materials And Methods}

\subsection{Materials}

70 patients of breast cancer were treated in hospital be-

Copyright () 2016 Yao Danyang et al.

doi: $10.18686 /$ jn.v5i2.12

Received: June 28, 2016; Accepted: August 1, 2016; Published online: August 25, 2016

This is an open-access article distributed under the terms of the Creative Commons Attribution Unported License (http://creativecommons.org/ licenses/by-nc/4.0/), which permits unrestricted use, distribution, and reproduction in any medium, provided the original work is properly cited. ${ }^{\star}$ Corresponding author: Jilin Cancer Hospital, Changchun, Jilin 130021, China. E-mail: yaodanyang008@sina.com tween December 2012 to December 2014 were selected as follow: age between 34 to 65 years, mean $49.3 \pm 7.4$ years; 64 cases are married, 6 cases are divorced; 15 cases from university, 21 cases from high school, 17 cases from secondary school, 11 junior high school and 6 cases from elementary school. 70 patients were randomly divided into observation group and control group, each one with 35 cases. All patients' clinical information was complete, no significant difference in general, which were comparable ( $p$ $<0.05)$.

\subsection{Nursing}

\subsubsection{Control group}

Conventional breast cancer nursing care was provided to the control group during perioperative period.

\subsubsection{Observation group}

Conventional nursing care and personalized nursing intervention in perioperative of breast surgery are as follows:

(1) Health education: explaining to patients about the disease, features and counter measures, providing patients with the necessary medical assistance and care guide, such as disease prevention, dietary guidance and medication principles. 
(2) Psychological intervention: Informing the affect in treatment on the psychological state of patients from the perspective of psychological medicine, teaching patients how to regulate emotions and self-control, and to have a positive attitude to face treatment. Any progress and efforts in patients should be positively affirmed and encouraged to achieve the patient's sense of self-worth, to meet the difficulties and challenges with confidence in order to minimize the patient's concerns, and to eliminate their negative emotions [2].

(3) Perioperative nursing: 30min of equipment preparation and sterilization before operation. According to operations requirement, it requires a skilled staff in delivering dissecting forceps, scissors and hook, timely removal of blood, eschar and others instruments. After the surgery was completed, a thorough inventory of all the instruments and dressings needs to be done, wash through the surgical field via saline water, remove any blood clots, and disinfect the skin and sutured wounds. Meanwhile intraoperative continuous ECG monitoring closely recorded the progress of surgery, abnormal events timely treated, through psychological and emotional communication with patients, distract patients by using praise language stimulation in order to assist patients to overcome the pain.

(4) Family care: enhancing of communication between nurses and patients, to understand and meet the needs of patients. Families of patients should be guided for a better communication with the patients and being understandable of the patient's inner feelings, to help patients to get attention, meticulous care and love from family. Nurses can be accountable for their actions and use one-on-one instruction to help patients with postoperative rehabilitation exercise in hospital.

(5) Sleep guidance: respecting patients' sleeping habits, providing scientific and reasonable sleep patterns, nurses should assist patients to develop time tables for activities, rest, sleep, and urge them to schedule regular daily activities, such as regular breaks and sleeping, and regular sleeping and waking time.

(6) Safety nursing: creating a quiet and comfortable room environment, keeping sheets clean, neat and dry. Nurses should encourage patients to eat easily digested and high energy food to build up their strength. Vital signs, ECG and blood oxygen saturation were observed to prevent hypercapnia. Nurses should guide patients to inhale slowly and deeply through their nose, to sink the diaphragm and expand the chest cavity to maximum in order to relieve pain.

(7) Health education after discharged: Guiding of universal breast self-examination: observe the skin color and inset of nipple, put finger flatly with light pressure on the breast when lying down, check turn-by-turn for any lump from outside to the nipple. Put hand behind head and check again. Finally, gently squeeze the nipple with thumb and forefinger, and observe for discharge. If any abnormal condition is observed, quickly go to hospital for a checkup to early detect the recurrence symptoms. In the diet, maintain a balanced diet and frequent smaller meals. Patients should avoid spicy and salty food, and take food that contains high protein such as meat, fish, eggs and dairy foods to expedite the wound healing process. Patients can take more vegetables, fruits, oatmeal, brown rice and other foods high in fiber to avoid constipation. In postoperative activity, not suitable to use the affected side upper limb measured blood pressure and venous puncture, in order to prevent limb swelling, avoid skin damage and reduce the incidence of infection. Avoid using the affected side upper limb to move and lift heavy objects, maintain a good mood and regular life can help to speed up recovery process after surgery. Walking a short distance can promote blood circulation, digestion and absorption, according to individual physical strength, progressive increasing in activity. Avoid pregnancy in the next five years after surgery, since pregnancy often promotes breast cancer recurrence [3].

\subsection{Therapeutic evaluation criteria}

\subsubsection{Treatment compliance evaluation criteria}

Divided into three levels, (1) Good compliance: fully accepting doctors developing treatment plan and actively cooperating with the nursing to complete the whole treatment process; (2) Partial compliance: accepting parts of doctor's develop treatment options and some nursing measures; (3) Noncompliance: the patient does not understand the adverse reactions that occur, do not fit therapy and nursing care [4].

\subsubsection{Psychological status evaluation criteria}

By self-rating anxiety scale (SAS) and self-rating depression scale (SDS) to evaluate the psychological state of patients.

\subsection{Statistical methods}

All the data are analyze using SPSS 18.0 statistical software, measurement data using mean \pm standard deviation to express, with test, counting data using percentage (\%), said by $\mathrm{C}^{2}$ test, and a comparison between groups by $p<0.05$, with statistical significance.

\section{Results}

\subsection{Compliance comparison}

Observation group patient compliance were significantly better than the control group, a statistically significant difference between groups $(p<0.05)$ (Table 1).

\subsection{Comparison of SAS and SDS scores}

Observation group of SAS and SDS scores were significantly better than the control group, a statistically significant difference between groups $(p<0.05)$ (Table 2).

\section{Discussion}

The incidence of breast tumor is higher in women, mostly 
Table 1. Compliance comparison of two groups of patients before and after treatment (case\%).

\begin{tabular}{ccccc}
\multirow{2}{*}{ Group } & \multicolumn{2}{c}{ Observation group } & \multicolumn{2}{c}{ The control group } \\
\cline { 2 - 5 } & Before treatment & After treament & Before treatment & After treament \\
\hline Good compliance & $10(28.57)$ & $24(68.57)$ & $11(31.42)$ & $13(37.14)$ \\
Partial compliance & $15(42.85)$ & $9(25.71)$ & $15(42.85)$ & $16(45.71)$ \\
Noncompliance & $10(28.57)$ & $2(5.71)$ & $9(25.71)$ & $6(17.14)$ \\
\hline
\end{tabular}

Table 2. Comparison of SAS and SDS scores of two groups of patients before and after treatment $(\bar{x} \pm \mathrm{s})$.

\begin{tabular}{cccccc}
\multirow{2}{*}{ Group } & \multirow{2}{*}{ Cases } & \multicolumn{3}{c}{ SAS (scores) } & \multicolumn{3}{c}{ SDS (scores) } \\
\cline { 3 - 7 } & & Before treatment & After treament & Before treatment & After treament \\
\hline Observation group & 35 & $59.6 \pm 6.2$ & $45.0 \pm 3.1$ & $62.1 \pm 9.8$ & $44.7 \pm 6.4$ \\
Control group & 35 & $58.3 \pm 5.4$ & $53.1 \pm 7.9$ & $62.5 \pm 7.9$ & $53.5 \pm 8.0$ \\
\hline
\end{tabular}

benign, and it is a common female malignancy. Currently, the main method of treating breast cancer with surgery, to some extent, will cause the breast surface scarring or even the entire breast to be removed, which is bound to give patients a strong mental stimulation. Before surgery, most patients don't have the correct understanding of breast cancer surgery. They often appear pessimism, despair, fear, anxiety and other negative emotional, which seriously affects the health and quality of life of patients and their families. This is not a conducive environment for surgical treatment and after surgery recovery. With the changing pattern of modern nursing, personalized care model has become more widely used in clinical care. Nurses gradually take an important nursing care role when patients are in hospital [5].

Judging from the SAS and SDS findings before treatment, most patients with breast cancer have a severe psychological burden, therefore, it is necessary to provide regular professional care and personalized care simultaneously.

In recent years, the traditional medical model began to change to the biology-psychology-social medical model. More medical workers began to realize the treatment and rehabilitation role in promoting of personalized care for patients with breast cancer. From the large number of patients with breast cancer survey, anxiety and depression in patients is a common psychological reaction. Sensitivity to pain is also one of psychological problems in patients. These negative emotions can cause sympathetic nerve activity and neurotransmitter changes in the body, thus causing a series of physiological changes of heart, blood vessels and blood flow dynamics, resulting in patients with high blood pressure, rapid heart rate, rapid breathing, etc ${ }^{[6]}$.

Personalized care is the way which refers to health education and psychological intervention combined and evidence-based care throughout the treatment. Previous study had surveyed on quality of lives of 97 cases of breast cancer patients. The researcher found out that education, occupation type, health care, family support and drug reactions have a significant impact on the quality of life of patients. In conclusion, if the caregiver can develop targeted, per- sonalized care measures and give the patient an effective psychological intervention, it can significantly improve the quality of patient's life. From the results of this study, the observation group of patients with personalized care measures of treatment and care compliance was significantly higher $(p<0.05)$. The improvement of anxiety and depression in the observation group were better than those in the control group $(p<0.05)$. It shows that personalized care measures will enable patients to get a good mental state, improve patients' treatment compliance and reduce the unpleasant feelings of patients. This relatively good results are worthy for tremendous health care promotion.

\section{Conflicts of interest}

These authors have no conflicts of interest to declare.

\section{Authors' contributions}

These authors contributed equally to this work.

\section{References}

1. Yuan M, Liang S, Ma L. Research of nursing intervention on the compliance of chemotherapy in patients with initial breast cancer. Chin J Misdiagnostics. 2009;(26):65486549.

2. Zhang R, Liu Y, Liu L. Self-efficacy Influence Factor Analysis and Nursing Intervention Experience of Patients with Breast Cancer. Chin J Misdiagnostics. 2009;(26):63586359.

3. Hong S. Effects of individualized nursing intervention on emotion and quality of sleep in patients with breast cancer. Pract Prev Med. 2014;21(10):1237-1238.

4. Cao H, Tan H, Huang $\mathrm{W}$, et al. The correlation analysis between postoperative quality of life in patients with breast cancer and anxiety and depression. Mod Clin Nurs. 2009;8(7):1-3.

5. Lu P. Application of individual nursing patterns in nursing after breast cancer surgery. Jilin Med J. 2013;34(32):6869-6870.

6. Li J, Wang Z, Chen A. Effect of psychological intervention on anxiety and depression in patients with malignant cancer. J Clin Med Pract. 2010;14(2):11-12. 